\title{
Chemosensory Changes and Quality of Life in Patients Undergoing Hematopoietic Stem Cell Transplantation
}

\author{
Rania Abasaeed ${ }^{1}$, Susan E. Coldwell ${ }^{1}$, Michele E. Lloid ${ }^{1,2}$, Sohaib H. Soliman ${ }^{1}$, Paula C. \\ Macris $^{2}$, and Mark M. Schubert ${ }^{1,2}$ \\ ${ }^{1}$ University of Washington School of Dentistry, 1959 NE Pacific Street, Seattle, WA 98195 \\ ${ }^{2}$ Seattle Cancer Care Alliance, 825 Eastlake Ave E, Seattle, WA 98109
}

\begin{abstract}
Purpose - to assess magnitude and characteristics of changes in chemosensory function and quality of life (QOL) for patients receiving hematopoietic stem cell transplantation (HSCT).

Methods-Patients (aged 18 years and above) scheduled to undergo HSCT at the Seattle Cancer Care Alliance were tested for chemosensory function at three time points: pre-transplant (Baseline), $30+/-5$ days (Day 30) and $80+/-5$ days (Day 80) post HSCT. Gustatory function was assessed following procedures developed at the Monell-Jefferson Taste and Smell Clinic.

Olfactory testing was conducted using the National Institute of Health Toolbox Odor Identification test. QOL was also assessed.

Results-Twenty-nine patients were enrolled in the study between August 2014 and March 2015. Twenty-three patients were included in the analysis, with sixteen tested at all three time points (Baseline, Day 30 and Day 80). The primary finding is decreased taste sensitivity for 0.32 $\mathrm{M} \mathrm{NaCl}, 0.0056 \mathrm{M}$ citric acid and $0.018 \mathrm{M}$ citric acid on Day 30 following HSCT. Increased taste sensitivity for $0.32 \mathrm{M}$ sucrose at Day 30 was also observed. Taste sensitivity largely recovered by Day 80. Olfactory identification scores were unchanged from baseline to Day 30. QOL was reduced at Day 30, but was restored to an acceptable level of functioning and symptoms by Day 80. However, some areas remain impaired.
\end{abstract}

Conclusions-Alterations in taste perception were confirmed in the early post-transplant period. This was largely resolved within 2.5 months. No obvious impairments were observed in olfactory function. QOL improved by Day 80, though some oral symptoms lingered.

\section{Introduction}

Hematopoietic stem cell transplantation (HSCT) is widely used as a therapeutic treatment for patients with both malignant and non-malignant hematological diseases as well as congenital immune deficiencies [1]. HSCT patients undergo preparative conditioning therapy consisting of chemotherapy, chemotherapy combined with radiation, or radiation

Corresponding Author: Susan E. Coldwell, School of Dentistry, Box 356365, University of Washington, Seattle, WA 98195-6365, scoldwel@uw.edu, 206-616-3087, ORCiD 0000-0002-6587-9007.

Conflict of Interest

The authors declare no conflict of interest. 
alone to provide immune suppression and eliminate the maximum number of cancer cells with the least amount of toxicity [2]. Stem cell sources include peripheral blood stem cells, bone marrow transplant, and umbilical cord blood. Donor types include autologous (using the patient's own stem cells), allogeneic (using donor stem cells), and syngeneic (using cells from an identical twin). Conditioning regimens include myeloblative (bone marrow depleting) and non myeloblative (bone marrow sparing) reduced intensity conditioning regimens. The intensive (myeloblative) conditioning regimens are associated with significant side effects such as anorexia, nausea, vomiting, diarrhea, and mucositis in the acute phase of transplant [3]. Loss or change in taste perception is a frequent complaint among most patients undergoing HSCT, particularly the allogeneic type [4]. Taste alterations have a negative effect on the quality of life of HSCT patients, decreasing food enjoyment, and reducing their overall nutritional intake [5].

A limited number of studies have focused on timing of taste alterations among HSCT patients. Marinone and colleagues [6] observed late and selective taste dysfunction in allogeneic transplant patients. The researchers determined taste thresholds for four of the five basic tastes in patients following HSCT (eight autologous, fifteen allogeneic) and compared with taste thresholds determined for twenty healthy control subjects. Allogeneic HSCT recipients had significantly increased threshold for detecting salty and sour tastes. A recent study by Hull and colleagues [7] found reduction in taste function in $20 \%$ of survivors of allogeneic HSCT examined between 6 months and 6 years post-HSCT using a standardized questionnaire with no objective taste testing. In a survey study, initial posttransplant reports of taste intensity changes in 50 patients receiving allogeneic HSCT appeared to have recovered by $90-100$ days post-transplant [4].

Alterations in the sense of taste among HSCT patients have been associated with weight loss due to a decrease in overall food intake. Lenssen and colleagues [8] reported nutritional problems in $23 \%$ and weight loss in $28 \%$ of 192 patients in the first year after allogeneic bone marrow transplantation. A high prevalence of eating disorders (66\% at day 50 posttransplant) has been found among HSCT patients in a longitudinal study in the Netherlands [9]. Taste alterations were mentioned as one of the symptoms most strongly associated with eating difficulties among study participants. Taste alterations occurred in $61 \%$ of the patients at day 50 post-transplant and remained in $7 \%$ at day 350 post-transplant [9].

While there is much evidence of taste dysfunction in the period immediately following HSCT, there is no consensus as to the time period over which taste function recovers. This is partly because of differences in the taste testing methodologies as well as differences in study populations between earlier studies. Another issue is that the majority of the studies have been cross-sectional in nature. In order to fully understand the extent of taste dysfunction in patients undergoing HSCT, it is necessary to objectively measure taste perception both before and after HSCT. A prospective study would help to clarify the relationship between taste dysfunction and time.

Many prior studies have focused on assessing taste dysfunction in isolation. However, gustatory and olfactory signals both contribute to the perception of flavor and palatability of food. Dysfunction in either of these senses may change dietary habits. Therefore, gathering 
data on the impact of HSCT on both taste and smell function is important in understanding any impact on ingestive behavior. Finally, in order to best understand the impact of HSCT and the associated therapeutic benefit and side effect on patients, it is important to assess the Quality of life (QOL). Taste alterations have previously been shown to be negatively associated with some aspects of QOL for HSCT patients [4].

In the current study, we prospectively enrolled patients scheduled to receive HSCT, assessing them before and after transplant. We hypothesized that chemosensory function of HSCT patients would decline following conditioning and transplant, and that QOL scores would decline over the same period. We further predicted based on general observation that unfavorable alterations in QOL scores and chemosensory function would be transient and would recover by the end of the study timeframe.

\section{Materials and Methods}

\section{Participants}

Patients included in the study were scheduled to undergo HSCT at the Seattle Cancer Care Alliance (SCCA) (Seattle, Washington, USA). Patients receiving stems cells from any source were considered eligible for this study. All conditioning regimes and diagnoses were eligible. Study enrollment started in August 2014 and was completed in May 2015.

Eligible patients completed a routine pre-transplant examination at SCCA's Oral Medicine clinic. Potential participants were approached and informed of the study by the Oral Medicine clinic staff personnel during this examination. Patients were recruited into the study if they were older than 18 years, were able to eat and drink by mouth, and spoke and understood English. Also, patients must have been registered to receive myeloablative or nonmyeloblative conditioning therapy prior to HSCT. Patients were excluded if they had self-reported allergy to quinine or were hospitalized in-patients at the time of testing.

\section{Procedures}

All participants received full head and neck and intra oral examination and any significant dental and periodontal disease was eliminated or stabilized prior to transplant conditioning. Data were collected prior to HSCT in order to establish levels of function at baseline. Additional data were collected at $30(+/-5)$ and $80(+/-5)$ days following HSCT. At each time point patients were assessed for: (a) quality of life (b) olfactory identification ability and (c) gustatory sensitivity. Demographic and health status information was retrieved from the medical record. SCCA-Fred Hutchinson Cancer Research Center (FHCRC) Ethics Committee approved the protocol (FHCRC Data Repository protocol \#884). Written, informed consent was obtained from the participants.

\section{Quality of Life}

The European Organization for Research and Treatment of Cancer (EORTC) core questionnaire, the EORTC QLQ-C30 (version 3.0), is used to assess how patients with cancer have perceived their health-related quality of life during the past week [10]. It contains 30 items distributed over 5 functional scales (physical, role, cognitive, emotional, 
and social), 3 symptom scales (fatigue, pain, and nausea or vomiting), and 1 global healthstatus/quality of life scale. This questionnaire also contains 6 single items addressing further cancer symptoms (dyspnoea, appetite loss, insomnia, constipation, and diarrhea) and the financial impact of the disease. Each item has 4 response alternatives: 1) "Not at all", 2) "A little", 3) "Quite a bit", and 4) "Very much", except the global health-status/quality of life scale, which has the response alternatives based on a categorical scale ranging between 1) "Very poor" and 7) "Excellent". The QLQ-C30 has been tested and shown good validity and reliability $[11,12,13]$.

The EORTC Head and Neck module, the QLQ-H\&N35 (version 1.0), includes 35 items [14, 15]. The QLQ-H\&N35 has seven subscales: pain (4 questions), swallowing (5 questions), senses (1 question on taste and 1 on smell), speech (3 questions), social eating (4 questions), social contact (5 questions), and sexuality (2 questions). In addition, 11 individual topics are evaluated (dental problems, mouth opening, dry mouth, poor salivation, coughing, sense of illness, analgesic use, nutrition difficulties, gastric tube, and weight loss or gain). The time frame of the module is "during the past week". Items Number 1-30 are scored on four-point Likert-type categorical scales ("not at all," "a little," "quite a bit," "very much"). Items Number 31-35 have a "no/yes" response format. For both QOL modules, scores are transformed into 0 -to-100 scales, with a high score implying a high level of symptoms or problems.

\section{Olfactory Testing}

Olfactory testing was conducted using the National Institute of Health (NIH) Toolbox Odor Identification test [16]. This test assesses a person's ability to identify various odors and has been validated by Dalton and colleagues [17]. The selected odors are lemon, Play-doh®, chocolate, bubble gum, popcorn, coffee, smoke, natural gas, and flower. Scratch 'n' sniff cards are used to administer the odors. After scratching the cards one at a time, participants are asked to identify which of four pictures (correct plus three distractors), matches the odor they have just smelled. The test takes approximately 4 to 5 minutes to administer and is validated for ages $3-85$. The odor ID test is scored by summing the number of correct responses across the nine odor cards.

\section{Gustatory testing}

Gustatory testing closely followed the procedures of the Rapid Screening Taste Test developed at the Monell-Jefferson Taste and Smell Clinic [18], but modified to use the General Labeled Magnitude Scale (gLMS) as the response scale [19]. Participants rated the intensity of three different concentrations each of chemicals representing four of the five basic taste modalities (sweet, salty, sour, and bitter). Solutions were presented in one of two randomized orders (order A at baseline and Day 80, order B at Day 30). The sweet solutions were made of sucrose in the following molar $(\mathrm{M})$ concentrations: $0.1 \mathrm{M}, 0.32 \mathrm{M}$, and $1.0 \mathrm{M}$. The salty solutions were made from sodium chloride $(0.1 \mathrm{M}, 0.32 \mathrm{M}$, and $1.0 \mathrm{M})$. Sour solutions were made from citric acid $(0.0018 \mathrm{M}, 0.0056 \mathrm{M}, 0.018 \mathrm{M})$. Bitter solutions were made from quinine hydrochloride $(0.0001 \mathrm{M}, 0.00032 \mathrm{M}$, and $0.001 \mathrm{M})$. Distilled water was also administered once. Participants were presented with $10 \mathrm{ml}$ of each solution in a medicine cup. Participants sipped the entire amount of each solution, swished it around in 
the mouth, and then expectorated the solution into a cup. Participants rated the intensity of each taste on the gLMS. Taste intensity data were scored as distance in mm marked from the bottom of the $123 \mathrm{~mm}$ gLMS scale.

\section{Other variables}

Demographic information collected from the electronic health records of participants included age, sex, hematologic diagnosis, stem cell source, donor relationship, degree of Human Leukocyte Antigen match, history of prior transplant, and conditioning regimen (ORCA, University of Washington Medical Center, Seattle, WA USA; Powerchart, Cerner Corporation, Kansas City, MO USA).

\section{Analyses}

Statistical Analyses were performed for participants who completed assessments at both baseline and Day $30(n=23)$. Descriptive statistics were calculated for patient demographics, clinical diagnosis, types of conditioning, graft versus host disease (GVHD) prophylaxis, and QOL scales. Paired t-tests were used to test for differences in Odor ID scores from baseline to day 30. ANOVA's were used to assess for main effects of test time (baseline versus Day 30), solution concentration (low, medium, high), and for time by concentration interactions. When significant time by solution concentration interactions were observed, Newman-Keuls post-hoc testing was used to assess for changes in taste intensity at each solution concentration. A separate ANOVA was conducted for each of the four basic tastes. In order to assess whether observed changes in quality of life and chemosensory function recovered, a subgroup analysis was additionally conducted for the sixteen patients who completed Day 80 testing.

\section{Results}

Twenty-nine patients were enrolled in the study between August 2014 and March 2015. Of those, 23 patients were included in the analysis after exclusion of patients who did not complete the study or refused follow-up testing (e.g., due to being unwell at the time of assessment). Demographic and clinical characteristics of the 23 included patients are shown in the Table 1.

\section{Quality of Life}

Average scores (and standard error) for the EORTC QLQ-C30 \& EORTC QLQ - H\&N35 surveys are depicted in Tables 2 and 3 as well as Figure 1. The highest symptom scores and lowest function scores were experienced 30 days after HSCT. Thereafter, the levels of symptoms decreased and levels of functioning increased towards baseline levels by day 80 . Exceptions from this pattern were dry mouth, sticky saliva, and weight loss. These symptoms remained present at both post-HSCT time periods.

\section{Odor Identification}

At baseline, the mean Odor ID score for patients was 7.96 ( $\mathrm{SD}=1.10)$, compared with 7.78 ( $\mathrm{SD}=1.12)$ at Day $30(\mathrm{p}=0.56, \mathrm{NS})$. These scores are comparable to the mean score of 7.5 
$(\mathrm{SD}=1.38)$ reported for 3,508 community-dwelling adults tested in the NIH Toolbox norming study [20].

\section{Gustatory Testing}

Gustatory testing data for the 23 patients tested at Baseline and Day 30 are depicted in Figure 2. Taste intensity (as mm on the gLMS scale) is plotted at baseline and Day 30 for each concentration of solution (low, medium, high). For $\mathrm{NaCl}$, there was a significant effect of concentration $[\mathrm{F}(2,44)=99.24, \mathrm{p}<0.001]$ and a significant concentration by time interaction $[\mathrm{F}(2,44)=3.96, \mathrm{p}<0.05]$. The medium concentration of $\mathrm{NaCl}(0.32 \mathrm{M})$ was rated significantly lower on Day 30 compared with baseline (Newman-Keuls, $\mathrm{p}<0.02$ ).

On Day 30 we also observed decreased intensity ratings for $0.018 \mathrm{M}$ citric acid (high concentration) and $0.0056 \mathrm{M}$ citric acid (medium concentration) compared with baseline. [Main effect of concentration, $\mathrm{F}(2,44)=71.8, \mathrm{p}<0.0001$, main effect of time, $\mathrm{F}(1,22)=$ $24.8, \mathrm{p}<0.0001$, and a significant concentration by time interaction, $\mathrm{F}(2,44)=5.53, \mathrm{p}<$ 0.02 . Both middle $(\mathrm{p}<0.01)$ and high $(\mathrm{p}<0.001)$ concentrations were rated significantly lower at Day 30 (Newman-Keuls post hoc tests).]

For quinine, there was a significant main effect of solution concentration $[F(2,44)=57.6, p$ $<0.0001]$, but no main effect of time or any time by concentration interactions to indicate that quinine intensity changed following HSCT.

For sucrose there was a significant main effect of concentration $F(2,44)=69.3, p<0.0001$ and a concentration by time interaction $F(2,44)=5.39, p<0.01$. The medium concentration of sucrose $(0.32 \mathrm{M})$ was significantly higher at Day 30 than at baseline (Newman-Keuls, $\mathrm{p}<$ $0.01)$.

Figure 3 depicts taste intensity data for the subgroup of 16 patients completing the study through Day 80. Intensity ratings differed by solution concentration for all four taste qualities [quinine, $\mathrm{F}(2,24)=36.2, \mathrm{p}<0.001 ; \mathrm{NaCl}, \mathrm{F}(2,22)=85.9, \mathrm{p}<0.001$; citric acid, $\mathrm{F}(2,24)=64.8, \mathrm{p}<0.001 ;$ sucrose, $\mathrm{F}(2,24)=48.7, \mathrm{p}<0.001]$. However, for these 16 patients, only intensity ratings for sucrose had a significant solution concentration by time interaction $[F(4,48)=3.91, p<0.01]$. Newman-Keuls post hoc tests confirmed that intensity ratings for the medium concentration of sucrose were elevated at Day 30 relative to baseline and Day 80.

\section{Discussion}

The past few decades witnessed a rise in HSCT procedures with improved outcomes and subsequent increases in survival rates among patients with different sets of conditions. Chemosensory changes are side effects of HSCT that are commonly reported by patients and observed by medical personnel. These changes are thought to negatively affect quality of life, nutrition status, and overall recovery. To the best of our knowledge, this study is the first to objectively assess both olfactory and gustatory function in HSCT patients prior to their transplant and at two time intervals after HSCT. The study also combined self-assessment of taste and smell with more objective chemosensory test procedures. 
We assessed patient quality of life using the EORTC QLQ-C30 and EORTC QLQ - H\&N35. The EORTC QLQ-C30 was also employed by Epstein and colleagues [4] at Day 90-100 following HSCT in a study of 50 patients receiving high-dose chemotherapy and allogeneic transplant. Compared with the Day 90-100 patients in the Epstein study, at Day 30 our patients had overall higher Physical and Role functional scale scores. However, both these domains declined from baseline to Day 30. Social functioning was comparable to that observed in the Epstein study. However, social functioning was low from start, and even though it improved over time, it was still impaired 80 days following HSCT. One reason may be that long hospitalization periods and frequent visits to outpatient clinics after HSCT limit patient's social life.

Symptoms related to the mouth and the digestive system such as dry mouth, appetite loss, and nausea/vomiting dramatically and predictably increased from baseline to Day 30 following HSCT. These symptoms decreased over next several months, except for dry mouth (xerostomia) and thick sticky saliva, which remained elevated on Day 80. This finding is consistent with results from Andresson and colleagues [21], who reported the persistence of mouth dryness in 25 patients undergoing myeloablative conditioning one year after HSCT. Despite the continued reports of dry mouth and sticky saliva, patients reported improvements in problems with their taste and smell. While senses problems scores remained elevated over baseline at Day 80, they were greatly reduced from Day 30 symptom reports.

We used objective tests of gustatory and olfactory function to confirm and augment the questionnaire data. Objective assessment of olfactory function following HSCT showed no change from baseline. Olfactory performance at both baseline and Day 30 was consistent with the national norms established in the NIH Toolbox norming study [20]. While these findings indicate that overall olfactory function stays intact through HSCT treatment, the methodology we employed was designed to detect impairment of function only, and would miss any hypersensitivity to odors brought on by HSCT treatment.

In assessing gustatory function, our results revealed normal upward trends in taste intensity scores as the concentration of chemicals (sucrose, citric acid, quinine and $\mathrm{NaCl}$ ) increased, indicating proper gustatory function in relation to differentiating between different chemical concentrations. Nevertheless, our results showed changes in taste intensity scores on Day 30 and Day 80 following HSCT, which varied by chemical and concentration. These longitudinal observations are largely consistent with cross-sectional studies of taste issues following HSCT previously reported in the literature.

In concordance with Marinone and colleagues [6], we observed decreased intensity ratings for some concentrations of citric acid and $\mathrm{NaCl}$ on Day 30 (hypogeusia). Marinone and colleagues [6] reported that thresholds for sour were significantly increased (hypogeusia) in 15 adult patients $4-51$ months after HSCT compared with those in 20 healthy subjects. Similarly, two studies report elevated detection thresholds (hypogeuesia) for $\mathrm{NaCl}$ after HSCT [6, 22]. 
In contrast, we observed heightened intensity ratings (hypergeusia) for $0.32 \mathrm{M}$ sucrose at Day 30, which returned to baseline levels by Day 80. Boer and colleagues [23] stated that 15 of 61 patients in their study reported subjective increases in taste perception following HSCT, whereas 22 reported decreases in taste perception. As in our study, Boer and colleagues found differences in perceived sucrose intensity over time, with those patients who more recently experienced HSCT reporting higher sucrose intensities than those further from transplant [23].

Taste receptor cells are particularly sensitive to cytotoxic antineoplastic treatment due to their high rate of cell turnover, which is believed to be the basis of taste changes during chemotherapy and radiotherapy cancer treatment [24]. Antineoplastic drugs may also affect taste by disrupting taste sensation conduction or by inhibiting synaptic reuptake [25]. Medications used by patients may also be expressed in saliva and thus directly stimulate or inhibit taste detection at the lingual surface [26]. Another plausible explanation for alterations of taste perception is the reduction in salivary flow in HSCT patients. It is known that reduced salivary flow (hyposalivation) is associated with alterations in oral flora constituents and that may affect the sense of taste [27]. However, the exact mechanism of how salivary composition affects taste perception is still under investigation [28, 29]. The observation that HSCT differentially impacts perception of different taste qualities suggests that the cellular/neural mechanisms involved in processing the various gustatory qualities are differentially sensitive to disruption by HSCT, recover at different rates, or both.

\section{Limitations}

Patients in our study reported experiencing dry mouth, however these symptoms appeared to be dissociated from taste issues, which improved before any reported reduction in dry mouth. Objective measures of salivary flow were not done, and would be required to better assess the relationship between taste dysfunction and salivary flow in HSCT patients. Furthermore, we did not record data on the presence of mucositis or oral candidiasis, both of which may affect gustatory function. A further limitation of the study was our small sample size. Only 16 of 23 patients were available to continue the study through Day 80. Our conclusions on return to function are thus based on a subset of an already moderately-sized patient population.

\section{Future research}

Further work is needed to understand the degree to which the relatively small and specific changes in taste perception observed with HSCT treatment in this study impact nutritional intake. Food preferences and appetite are both influenced by factors other than direct changes in taste perception. For example, learned associations between flavor and nausea/ vomiting readily condition food aversions in cancer patients [30]. It is unclear the degree to which the subtle changes in taste perception observed here directly impact enjoyment of food.

The physiological mechanisms involved in sour taste transduction are less understood than are those involved in the remaining four basic tastes. The relative susceptibility of the sour taste of citric acid to disruption by HSCT compared with the three other taste modalities 
tested is interesting. Further work is needed to determine if these observations generalize to other sour tastes or rather is an impact limited to citric acid.

\section{Acknowledgments}

This work was supported by funding from the University of Washington Department of Oral Medicine, the Institute for Translational Health Sciences TL1 TR000422 (S. Soliman) and the Washington Dental Service Endowed Professorship (S. Coldwell). The authors would like to thank Sumia Dakhil for her assistance with data entry.

\section{References}

1. Copelan EA. Hematopoietic stem-cell transplantation. N Engl J Med. 2006; 354:1813-1826. [PubMed: 16641398]

2. Hamadani M, Mohty M, Kharfan-Dabaja M. Reduced intensity conditioning allogeneic HCT in adults with acute myeloid leukemia. Cancer Control. 2011 Oct; 18(4):237-245. [PubMed: 21976242]

3. Gratwohl A, Baldomero H, Aljurf M, Pasquini MC, Bouzas LF, Yoshimi A, et al. Hematopoietic stem cell transplantation: a global perspective. J Am Med Assoc. 2010; 303:1617-162.

4. Epstein JB, Phillips N, Parry J, et al. Quality of life, taste, olfactory and oral function following high-dose chemotherapy and allogeneic hematopoietic cell transplantation. Bone Marrow Transplant. 2002; 30:785-792. [PubMed: 12439702]

5. McIssac T. MS. Thesis. University of Alberta; Canada: 2008. Chemosensory Dysfunction in Advanced Cancer Patients.

6. Marinone MG, Rizzoni D, Ferremi P, et al. Late taste disorders in bone marrow transplantation: clinical evaluation with taste solutions in autologous and allogeneic bone marrow recipients. Haematologica. 1991; 76:519-522. [PubMed: 1820992]

7. Hull KM, Kerridge I, Schifter M. Long-term oral complications of allogeneic haematopoietic SCT. Bone Marrow Transplant. 2012; 47(2):265-70. [PubMed: 21441960]

8. Lenssen P, Sherry MA, Cheney CL, et al. Prevalence of nutrition-related problems among long-term survivors of allogeneic marrow transplantation. J Am Diet Assoc. 1990; 90:835-842. [PubMed: 2345257]

9. Iestra JA, Fibbe WE, Zwinderman AH, Van Staveren WA, Kromhout D. Body weight recovery, eating difficulties and compliance with dietary advice in the first year after stem cell transplantation: a prospective study. Bone Marrow Transplantation. 2002; 29:417-424. [PubMed: 11919732]

10. http://www.eortc.be/qol/files/SCManualQLQ-C30.pdf

11. Aaronson NK, Ahmedzai S, Bergman B, et al. The European Organization for Research and Treatment of Cancer QLQ-C30: a quality-of-life instrument for use in international clinical trials in oncology. J Natl Cancer Inst. 1993; 85:3665-376.

12. Groenvold M, Klee MC, Sprangers MA, Aaronson NK. Validation of the EORTC QLQ-C30 quality of life questionnaire through combined qualitative and quantative assessment of patientobserver agreement. J Clin Epidemiol. 1997; 50:441-450. [PubMed: 9179103]

13. Hjermstad MJ, Fossa SD, Bjordal K, Kaasa S. Test/retest study of the European Organization for Research and Treatment of Cancer Core Quality-of-Life Questionnaire. J Clin Oncol. 1995; 13:1249-1254. [PubMed: 7738629]

14. http://groups.eortc.be/qol/sites/default/files/img/specimen_for_printing_hn35.pdf

15. Bjordal K, Hammerlid E, Ahlner-Elmqvist M, de Graeff A, Boysen M, Evensen JF, Biörklund A, de Leeuw JR, Fayers PM, Jannert M, Westin T, Kaasa S. Quality of life in head and neck cancer patients: validation of the European Organization for Research and Treatment of Cancer Quality of Life Questionnaire-H\&N35. J Clin Oncol. 1999; 14:1008-1019.

16. nihtoolbox.org

17. Dalton P1, Doty RL, Murphy C, Frank R, Hoffman HJ, Maute C, Kallen MA, Slotkin J. Olfactory assessment using the NIH Toolbox. Neurology. 2013 Mar 12; 80(11 Suppl 3):S32-6. DOI: 10.1212/WNL.0b013e3182872eb4 [PubMed: 23479541] 
18. Pribitkin E, Rosenthal MD, Cowart BJ. Prevalence and causes of severe taste loss in a chemosensory clinic population. Ann Otol Rhino Laryngol. 2003 Nov; 112(11):971-978.

19. Bartoshuk LM. The psychophysics of taste. Am J Clin Nutr. 1978; 31:1068-1077. [PubMed: 352127]

20. https://dataverse.harvard.edu/dataset.xhtml?persistentId=doi:10.7910/DVN/FF4DI7

21. Andersson I, Ahlberg K, Stockelberg D, Brune M, Persson L-O. Heatlh-Related Quality of life in patients undergoing allogenic cell transplantation after reduced intensity conditioning versus myeloablative conditioning. Cancer Nursing. 2009; 32(4):325-334. [PubMed: 19444087]

22. Mattsson T, Arvidson K, Heimdahl A, et al. Alterations in taste acuity associated with allogeneic bone marrow transplantation. J Oral Pathol Med. 1992; 21:33-37. [PubMed: 1593493]

23. Boer CC, Correa ME, Miranda EC, de Souza CA. Taste disorders and oral evaluation in patients undergoing allogeneic hematopoietic SCT. Bone Marrow Transplant. 2010 Apr; 45(4):705-11. Epub 2009 Sep 21. [PubMed: 19767788]

24. Hovan AJ, Williams PM, Stevenson-Moore P, Wahlin YB, Ohrn KE, Elting LS, et al. A systemic review of dysgeusia induced by cancer therapies. Support Care Cancer. 2010; 18(8):1081-1087. [PubMed: 20495984]

25. Doty RL, Bromley SM. Effects of drugs on olfaction and taste. Otolaryngol Clin North Am. 2004; 37(6):1229-54. DOI: 10.1016/j.otc.2004.05.002 [PubMed: 15563912]

26. Zervakis J, Graham BG, Schiffman SS. Taste effects of lingual application of cardiovascular medications. Physiol Behav. 2000 Jan; 68(3):405-413. [PubMed: 10716552]

27. Fernando I, Patel T, Billingham L, et al. The effect of head and neck irradiation on taste dyfunction: a prospective study. Clin Oncol. 1995; 7:173-178.

28. Fábián TK, Beck A, Fejérdy P, Hermann P, Fábi900E1;n G. Molecular Mechanisms of Taste Recognition: Considerations about the Role of Saliva. International Journal of Molecular Sciences. 2015; 16(3):5945-5974. DOI: 10.3390/ijms16035945 [PubMed: 25782158]

29. Vissink A, Burlage FR, Spijkervet FK, Jansma J, Coppes RP. Prevention and treatment of the consequences of head and neck radiotherapy. Crit Rev Oral Biol Med. 2003; 14:213-225. [PubMed: 12799324]

30. Bernstein IL. Learned food aversions in the progression of cancer and its treatment. Ann NY Acad Sci. 1985; 443:365-380. [PubMed: 2990286] 


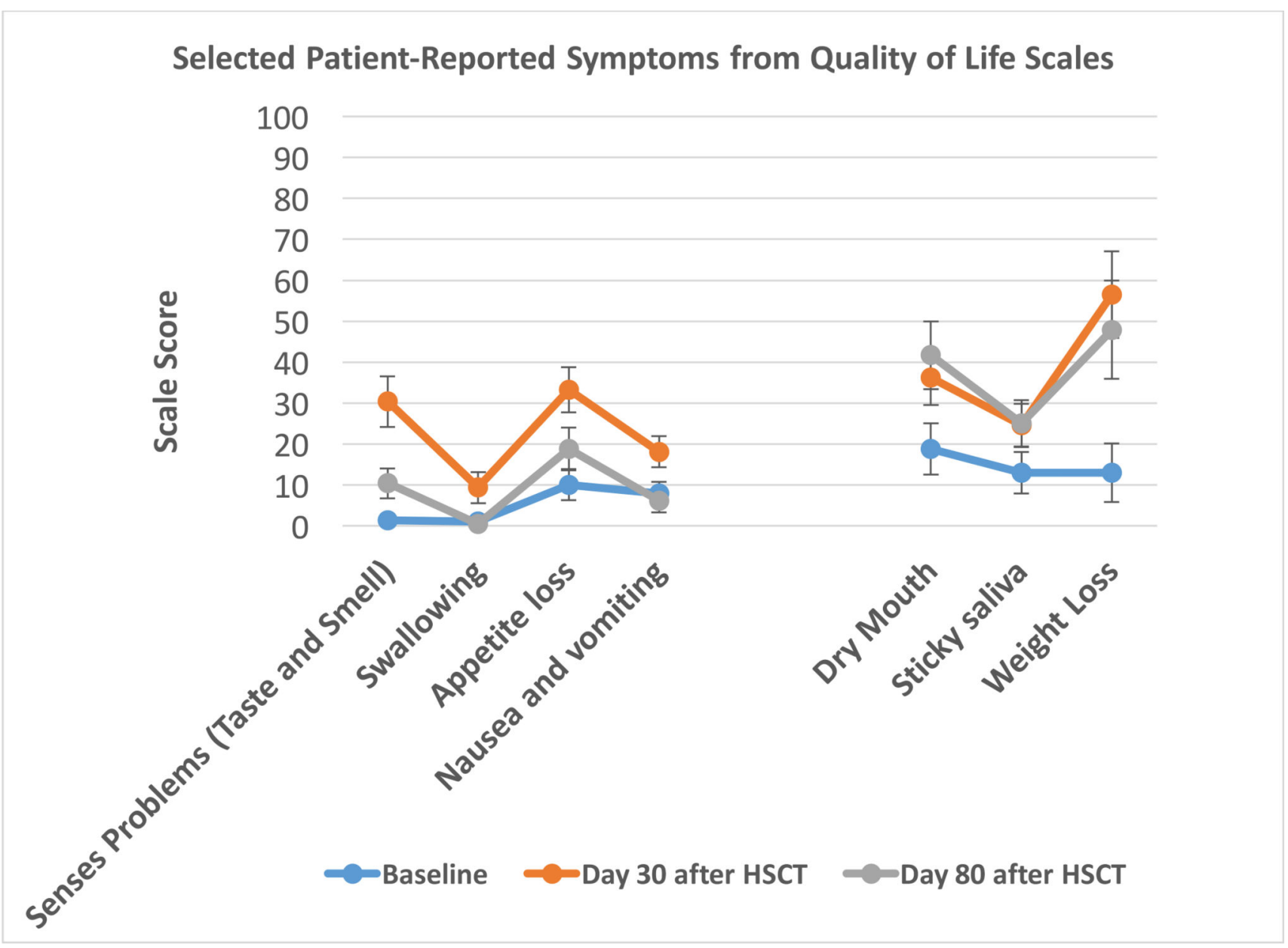

Figure 1.

Quality of life symptom scale scores as measured using the European Organization for Research and Treatment of Cancer (EORTC) core questionnaire, the EORTC QLQ-C30 (version 3.0), and the Head and Neck module of the EORTC, the QLQ-H\&N35 (version 1.0). Baseline and Day 30 scores are averages from the 23 patients completing the testing at both time points. Day 80 scores are averages of the 16 patients completing testing through Day 80. Scores range from 0 to 100 , with higher scores representing higher levels of symptoms. Whiskers depict standard error of each mean score. Symptoms depicted on the left of the graph were increased 30 days after HSCT, but improved by 80 days. Symptoms depicted on the right of the graph remained elevated 80 days after HSCT. 


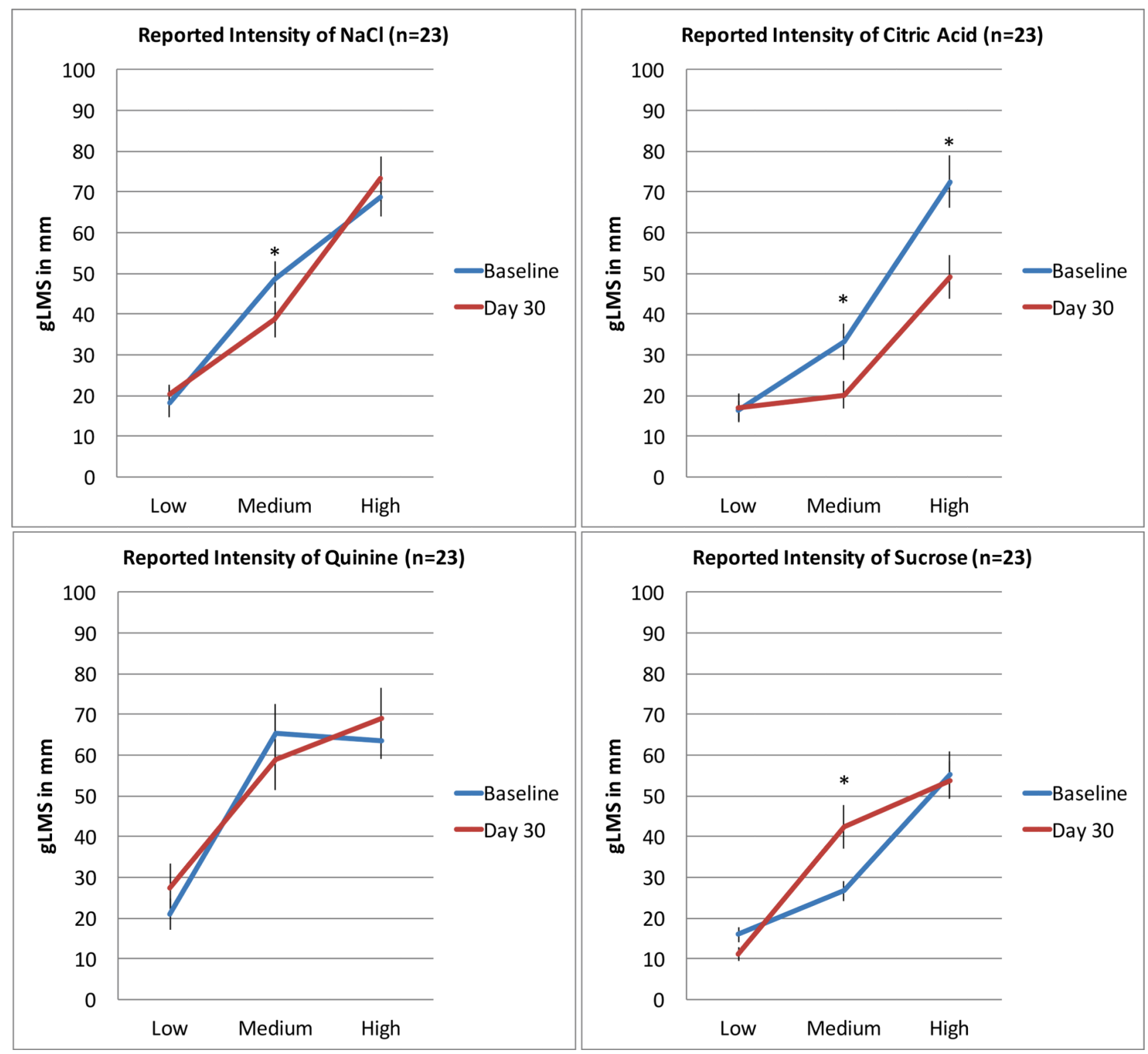

Figure 2.

Taste intensity scores measured in mm on the general Labeled Magnitude Scale (gLMS) for low, medium, and high concentrations of $\mathrm{NaCl}$ (upper left), citric acid (upper right), quinine (lower left) and sucrose (lower right). Average scores for 23 patients tested prior to transplant (baseline) and 30 (+/- 5) days after transplant (Day 30) are depicted. Error bars represent standard error of the mean. *Indicates a significant difference $(p<0.05)$ between baseline and Day 30 by Newman-Keuls post-hoc test. 


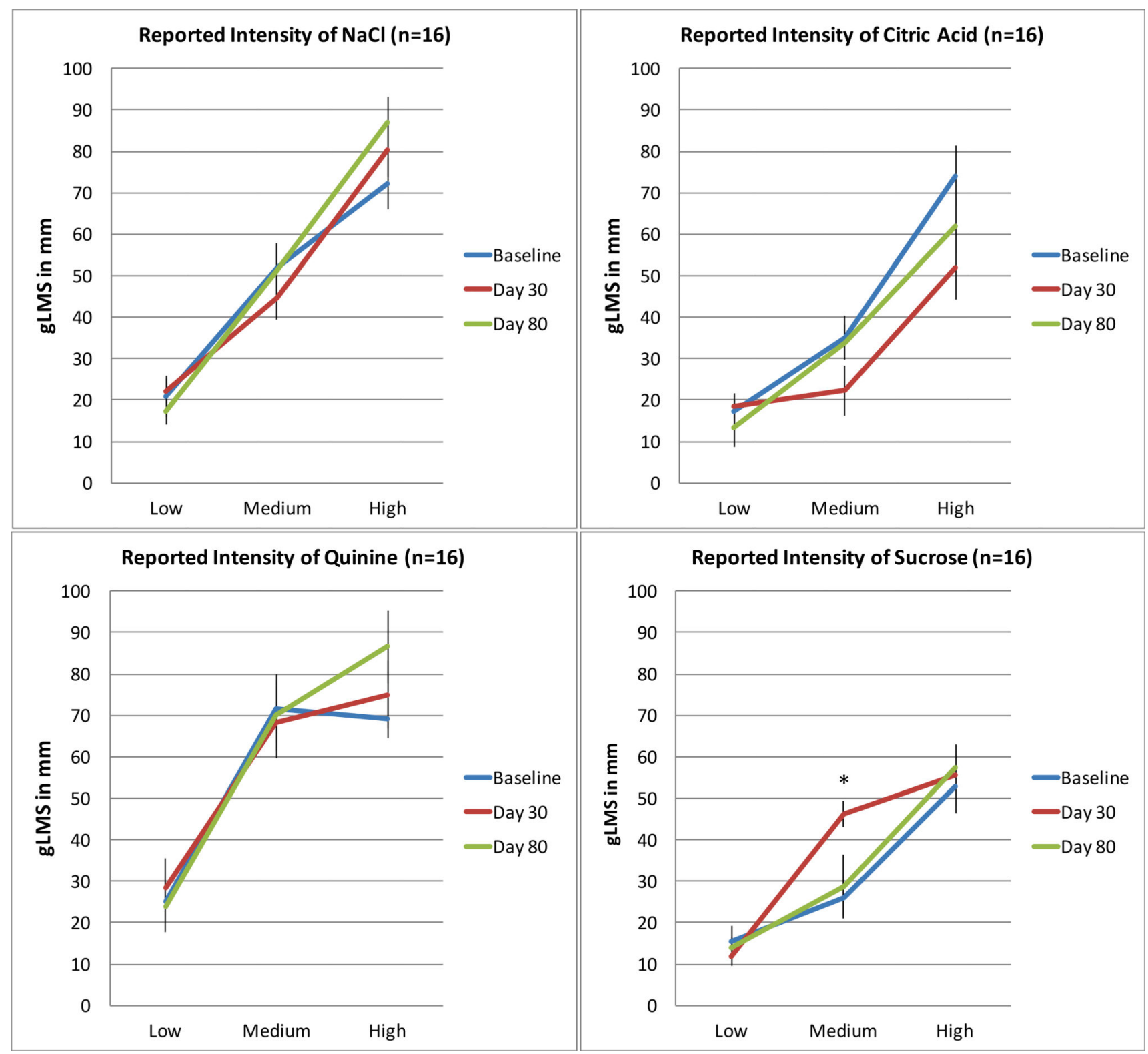

Figure 3.

Taste intensity scores measured in mm on the general Labeled Magnitude Scale (gLMS) for low, medium, and high concentrations of $\mathrm{NaCl}$ (upper left), citric acid (upper right), quinine (lower left) and sucrose (lower right). Average scores for 16 patients tested prior to transplant (baseline), 30 (+/- 5) days after transplant (Day 30), and 80 (+/- 5) days after transplant (Day 80) are depicted. Error bars represent standard error of the mean. *Indicates a significant difference $(\mathrm{p}<0.05)$ between Day 30 and baseline and Day 30 and Day 80 by Newman-Keuls post-hoc tests. 


\section{Table 1}

\section{Patient Demographics}

\begin{tabular}{|c|c|c|}
\hline & & $\mathrm{n}(\%)$ \\
\hline \multirow[t]{2}{*}{ Sex } & Male & $9(39)$ \\
\hline & Female & $14(61)$ \\
\hline \multirow[t]{3}{*}{ Age at transplantation } & Mean & 50 \\
\hline & Median & 49 \\
\hline & Range & 47 \\
\hline \multirow[t]{10}{*}{ Diagnosis } & AML & $6(26)$ \\
\hline & MM & $5(22)$ \\
\hline & MDS & $3(13)$ \\
\hline & ALL & $3(13)$ \\
\hline & Mantle Cell, Non-Hodgkin's Lymphoma & $1(4)$ \\
\hline & Diffuse Large B-Cell Lymphoma & 1(4) \\
\hline & B-Cell Follicular Lymphoma Stage III & 1(4) \\
\hline & Myelofibrosis & 1(4) \\
\hline & Blastic plasmacytoid dendritic cell neoplasm & 1(4) \\
\hline & CML & 1(4) \\
\hline \multirow[t]{2}{*}{ Transplant Type } & Autologus & $7(30)$ \\
\hline & Allogeneic & $16(69)$ \\
\hline \multirow[t]{2}{*}{ Conditioning Protocol } & Myeloablative & $20(87)$ \\
\hline & RIC & $3(13)$ \\
\hline \multirow[t]{2}{*}{ Source of stem cells } & PBSC & $20(87)$ \\
\hline & Cord blood transplant & 2(9) \\
\hline \multirow[t]{4}{*}{ Donor } & MRD & $5(22)$ \\
\hline & MURD & $6(26)$ \\
\hline & MMRD & $1(4)$ \\
\hline & MMURD & $3(13)$ \\
\hline \multirow[t]{10}{*}{ Conditioning protocols } & Myeloablative $\mathrm{Bu} / \mathrm{Cy}$ & $5(22)$ \\
\hline & Myeloablative MEL & $4(17)$ \\
\hline & Myeloablative Flu, treosulfan & $3(13)$ \\
\hline & Myeloablative TBI/Cy & $3(13)$ \\
\hline & Myeloablative BEAM & 2(9) \\
\hline & Nonmyeloablative Flu/Cy/TBI. & 2(9) \\
\hline & Myeloablative Radiolabeled Anti-CD45/TBI/Flu & 1(4) \\
\hline & Myeloablative Cy/TBI/ etoposide (VP16) & 1(4) \\
\hline & Myeloablative Flu/Cy/TBI & 1(4) \\
\hline & Nonmyeloablative Y-90 DOTA Bioten/Flu/TBI & 1(4) \\
\hline \multirow[t]{2}{*}{ GVHD Prophylaxis protocol } & Cyclosporine, Mycophenolate Mofetil & $6(26)$ \\
\hline & Methotrexate, Tacrolimus & $4(17)$ \\
\hline
\end{tabular}




\begin{tabular}{|l|l|c|}
\hline & & $\mathbf{n}(\%)$ \\
\hline & Cyclosporine, Methotrexate & $2(9)$ \\
\hline & Methotrexate, Tacrolimus, Abatacept & $2(9)$ \\
\hline & Mycophenolate Mofetil, Tacrolimus & $1(4)$ \\
\hline GVHD & Cyclophosphamide, Mycophenolate Mofetil, Tacrolimus & $1(4)$ \\
\hline & Acute GVHD (GI +/or Skin) & $14(61)$ \\
\hline & Acute Pseudo GVHD & $1(4)$ \\
\hline & Chronic skin GVHD at departure & $1(4)$ \\
\hline & Symptoms suggestive of oral GVHD at departure & $3(13)$ \\
\hline
\end{tabular}

$\mathrm{ALL}=$ acute lymphoblastic leukemia; $\mathrm{AML}=$ acute myelogenous leukemia; $\mathrm{CLL}=$ chronic lymphoblastic leukemia; $\mathrm{CML}=$ chronic myelogenous leukemia; $\mathrm{CMML}=$ chronic myelomonocytic leukemia; $\mathrm{MDS}=$ myelodysplastic syndrome; $\mathrm{MMRD}=$ mismatched related donor; $\mathrm{MMURD}=$ mismatched unrelated donor; $\mathrm{MRD}=$ matched related donor; $\mathrm{MURD}=$ matched unrelated donor; $\mathrm{PBSC}=$ peripheral blood stem cell; $\mathrm{RIC}=$ reduced intensity conditioning; $\mathrm{TBI}=$ total body irradiation; $\mathrm{Bu}=$ busulfan; $\mathrm{Cy}=$ cyclophosphamide; $\mathrm{MEL}=$ melphalan; Flu= fludarabine; $\mathrm{Beam}=$ carmustine, etoposide, cytarabine, melphalan 


\section{Table 2}

Average (standard error) scores on the functional and symptom scales of European Organization for Research and Treatment of Cancer (EORTC) core questionnaire, the EORTC QLQ-C30 (version 3.0), are shown in Table 2. Scores from the 23 patients completing the questionnaire prior to transplant (Baseline) and $30(+/-5)$ days (Day 30) after transplant are depicted in the middle two columns. Scores from the 16 patients who also also completed the questionnaire $80(+/-5)$ days after transplant (Day 80) are shown in the right-hand column.

\begin{tabular}{|c|c|c|c|}
\hline & $\begin{array}{c}\text { Baseline } \\
\mathbf{n}=\mathbf{2 3}\end{array}$ & $\begin{array}{c}\text { Day } 30 \text { after HSCT } \\
\mathbf{n}=\mathbf{2 3}\end{array}$ & $\begin{array}{c}\text { Day } 80 \text { after HSCT } \\
n=16\end{array}$ \\
\hline \multicolumn{4}{|l|}{ Functional scales ${ }^{a}$} \\
\hline Physical & $88.0(2.5)$ & $78.6(4.0)$ & $84.7(2.4)$ \\
\hline Role & $68.1(3.1)$ & $63.8(5.2)$ & $70.8(6.0)$ \\
\hline Emotional & $81.5(3.1)$ & $82.6(3.3)$ & $83.3(6.0)$ \\
\hline Cognitive & $79.0(4.1)$ & $76.8(5.2)$ & $87.5(4.7)$ \\
\hline Social & $58.0(5.0)$ & $56.5(5.2)$ & $65.6(7.2)$ \\
\hline Global QOL & $68.1(3.1)$ & $57.6(4.0)$ & $62.0(6.1)$ \\
\hline \multicolumn{4}{|l|}{ Symptom scales $b$} \\
\hline Appetite loss & $10.1(3.8)$ & $33.3(5.5)$ & $18.8(5.2)$ \\
\hline Fatigue & $26.9(3.0)$ & $42.0(4.5)$ & $34.6(4.0)$ \\
\hline Pain & $14.5(3.2)$ & $15.2(4.3)$ & $19.8(4.6)$ \\
\hline Dyspnea & $13.1(4.1)$ & $8.7(3.8)$ & $18.7(4.3)$ \\
\hline Insomnia & $21.7(4.9)$ & $33.3(6.3)$ & $35.4(6.4)$ \\
\hline Constipation & $14.5(4.6)$ & $11.6(5.4)$ & $12.5(5.9)$ \\
\hline Diarrhea & $14.5(5.5)$ & $23.2(6.1)$ & $14.6(6.1)$ \\
\hline Nausea and vomiting & $8.0(2.7)$ & $18.1(3.8)$ & $6.2(2.9)$ \\
\hline Financial Difficulties & $40.6(6.6)$ & $30.4(5.1)$ & $35.4(7.1)$ \\
\hline
\end{tabular}




\section{Table 3}

Average (standard error) scores on the functional and symptom scales the Head and Neck module of the EORTC, the QLQ-H\&N35 (version 1.0). Scores from the 23 patients completing the questionnaire prior to transplant (Baseline) and 30 (+/-5) days (Day 30) after transplant are depicted in the middle two columns. Scores from the 16 patients who also completed the questionnaire 80 (+/-5) days after transplant (Day 80) are shown in the right-hand column.

\begin{tabular}{|c|c|c|c|}
\hline & $\underset{\mathbf{n}=\mathbf{2 3}}{\text { Baseline }}$ & $\begin{array}{c}\text { Day } 30 \text { after } \\
\text { WHSCT } \\
n=23\end{array}$ & $\begin{array}{c}\text { Day } 80 \text { after } \\
\text { HSCT } \\
\text { n=16 }\end{array}$ \\
\hline \multicolumn{4}{|l|}{ Symptom scales $b$} \\
\hline Pain H\&N & $3.6(1.6)$ & $7.6(3.8)$ & $1.0(0.7)$ \\
\hline Swallowing & $1.1(0.6)$ & $9.4(3.8)$ & $0.5(0.5)$ \\
\hline Dry Mouth & $18.8(6.2)$ & $36.2(6.6)$ & $41.7(8.3)$ \\
\hline Sticky saliva & $13.0(5.0)$ & $24.6(5.2)$ & $25.0(5.7)$ \\
\hline Senses Problems (Taste and Smell) & $1.4(1.0)$ & $30.4(6.2)$ & $10.4(3.7)$ \\
\hline Speech problems & $3.0(0.9)$ & $7.3(2.4)$ & $2.0(1.5)$ \\
\hline Social eating trouble & $2.5(1.1)$ & $16.6(3.8)$ & $5.7(2.4)$ \\
\hline Social contact trouble & $9.3(3.2)$ & $16.2(3.6)$ & $13.7(4.6)$ \\
\hline Less sexuality & $28.2(6.4)$ & $44.9(6.9)$ & $46.7(9.5)$ \\
\hline Teeth & $7.2(3.6)$ & $4.3(2.4)$ & $6.24(3.4)$ \\
\hline Opening mouth & $2.9(2.0)$ & $10.1(3.9)$ & $4.2(4.2)$ \\
\hline Coughing & $14.5(3.5)$ & $20.3(5.0)$ & $8.3(3.7)$ \\
\hline Felt ill & $2.9(2.9)$ & $21.7(4.9)$ & $4.2(2.8)$ \\
\hline Pain killers & $21.7(8.8)$ & $26.1(9.4)$ & $37.5(12.5)$ \\
\hline Nutritional Supplements & $17.4(8.1)$ & $30.4(9.8)$ & $25.0(11.2)$ \\
\hline Feeding Tube & $0(0)$ & $0(0)$ & $0(0)$ \\
\hline Weight Loss & $13.0(7.2)$ & $56.5(10.6)$ & $47.9(12.0)$ \\
\hline Weight Gain & $34.8(10.1)$ & $30.4(9.8)$ & $18.8(10.1)$ \\
\hline
\end{tabular}

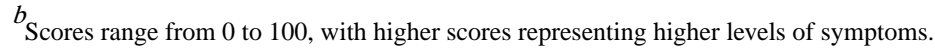

